\title{
Impact of intraoperative specimen mammography on margins in breast-conserving surgery
}

\author{
TOMOKA HISADA ${ }^{1}$, MASATAKA SAWAKI ${ }^{1}$, JUNKO ISHIGURO ${ }^{1}$, YAYOI ADACHI ${ }^{1}$, HARURU KOTANI ${ }^{1}$, \\ AKIYO YOSHIMURA ${ }^{1}$, MASAYA HATTORI $^{1}$, YASUSHI YATABE ${ }^{2}$ and HIROJI IWATA ${ }^{1}$ \\ Departments of ${ }^{1}$ Breast Oncology and ${ }^{2}$ Pathology and Molecular Diagnostics, \\ Aichi Cancer Center Hospital, Nagoya, Aichi 464-8681, Japan
}

Received January 29, 2016; Accepted May 9, 2016

DOI: $10.3892 / \mathrm{mco} .2016 .948$

\begin{abstract}
A positive resection margin is one of the most significant risk factors for local breast cancer recurrence following breast-conserving surgery (BCS). Intraoperative specimen mammography (SMMG) is routinely used to evaluate the surgical margin at our institution. The aim of the present study was to assess the adequacy of SMMG for margin assessment. The patient cohort included 174 women who underwent BCS in 2006. The sensitivity and specificity of SMMG were assessed by comparing the margins assessed by histological and radiological methods. It was also examined whether the rate of positive histological margins was decreased by re-excision following SMMG evaluation. A total of 23 false-negatives and 6 false-positives were determined by SMMG. The sensitivity and specificity of SMMG margin assessment for patients with primary breast cancer were 20.6 and $94.6 \%$, respectively. The positive predictive value was $50 \%$ and the negative predictive value was $82.2 \%$. A subgroup analysis revealed that the sensitivity and specificity of SMMG in cases with ductal carcinoma in situ (DCIS) were higher compared with those in invasive ductal carcinoma. Furthermore, the positive histological margin rate was not affected by re-excision. Although the general usefulness of intraoperative SMMG was not proven, this procedure may be useful in specific cases, particularly those with DCIS and those diagnosed by stereotactic biopsy. A prospective study with exact criteria and a standard procedure is required.
\end{abstract}

\section{Introduction}

The standard surgical treatments for breast cancer are breast-conserving surgery (BCS) and mastectomy. The essential purpose of BCS is to completely remove the cancer while

Correspondence to: Dr Masataka Sawaki, Department of Breast Oncology, Aichi Cancer Center Hospital, 1-1 Kanokoden, Chikusa-Ku, Nagoya, Aichi 464-8681, Japan

E-mail:m-sawaki@aichi-cc.jp

Key words: breast cancer, breast-conserving surgery, specimen mammography, intraoperative radiography, intraoperative mammography maintaining the cosmetic appearance of the breast, which is important to the patients. Previous studies have reported that BCS followed by radiation had a similar outcome to that of mastectomy in terms of mortality rate (1-5). However, a positive margin and young age $(<53-55)$ are significant risk factors for ipsilateral breast tumor recurrence (IBTR) (6-8). Moreover, a meta-analysis by the Early Breast Cancer Trialists' Collaborative Group showed that local recurrence affected survival rate (9). Recently, Moran et al (10) reported that the American Society of Radiation Oncology consensus guideline uses the results of a meta-analysis of margin width and IBTR. This guideline also indicated the optimal criteria for determining margin width in BCS. However, despite numerous previous studies, the criteria for intraoperative margin assessment in BCS have yet to be established. At our institution, intraoperative specimen digital mammography (SMMG) is used to assess the resection margins in all cases of BCS. The aim of the present study was to evaluate the usefulness of SMMG for achieving margin-free resection of breast tumors.

\section{Patients and methods}

Patients. A retrospective study of the medical records, pathological diagnosis and re-evaluation by SMMG of 426 breast cancer patients who underwent BCS at the Aichi Cancer Center Hospital (Nagoya, Japan) between January and December, 2006 was performed. All the patients had undergone MMG, ultrasonography (US) and magnetic resonance imaging (MRI) preoperatively. When the lesion was small and localized, BCS was suggested as an option to the patient. During the study period, a total of 174 patients underwent BCS. The surgical procedures were performed by 5 certified breast surgeons. SMMG was performed using the digital mammography unit Senographe 2000D ${ }^{\mathrm{TM}}$ (GE Healthcare Japan Corp., Tokyo, Japan), which had a pixel size of $100 \mu \mathrm{m}$. The display monitor had a resolution of 5 megapixels.

The study protocol was approved by our Institutional Review Board and written informed consent was obtained from all the patients.

Surgical procedure. BCS was performed concomitantly with sentinel lymph node biopsy or axillary lymph node dissection in all the patients. When scheduled, sentinel lymph node biopsy 
was performed at the beginning of the surgical procedure. Preoperative localization of the lesion was performed on the day prior to surgery by MMG, US and MRI. For non-palpable tumors and those undetectable by US, resection relied on hookwire-guided localization. Any localization detected by US was marked directly on the skin. For all patients, BCS was performed with at least a $2-\mathrm{cm}$ margin around the tumor. The pectoral fascia was resected. When the lesions were very close to the overlying skin, the skin was also resected.

The MMG device was placed in the room adjacent to the operating room where the resected specimen was collected and SMMG was performed. Images were immediately captured and stored in the hospital diagnostic imaging system. The surgeon quickly reviewed the digital images to assess the completeness of resection. If the resection was considered by the surgeon to be close to the margin, a re-excision was performed intraoperatively. The surgeon then assessed whether re-excision was complete. Intraoperative histological margin assessment was generally not performed.

SMMG evaluation. Two surgeons independently evaluated the SMMG findings. The classification of the radiographic evaluation of the resection margin was as follows: i) Negative, no cancer detected $<10 \mathrm{~mm}$ from the margin; ii) close, cancer detected within $5 \mathrm{~mm}$ from the margin; iii) positive, cancer detected $<5 \mathrm{~mm}$ from the margin; and iv) lesion undetected by SMMG. The results of the two surgeons were collectively classified as positive or negative. When the two evaluations did not agree, the more severe evaluation was selected (Table I).

Histopathological evaluation. The surgical specimens were prepared for histological analysis. The resected tissues were fixed in an adjustable mould (11). The tissue fixed using this method retains its polyhedral shape and pathologists are able to evaluate all aspects of the 5-mm blocks by hematoxylin and eosin staining. Using this procedure, all the surfaces of the specimen may be evaluated, i.e., using the perpendicular inked method and the tangential shaved method $(12,13)$. The margins were measured grossly and microscopically by determining the presence of cancer cells at a fixed distance from the cut edge. The distance between the margin of the tumor and the edge of the specimen was measured by the pathologists. A clear margin was defined as $>5 \mathrm{~mm}$. These criteria also apply to ductal carcinoma in situ (DCIS).

The diagnostic reliability of SMMG was evaluated by comparison with radiographic and histological diagnoses. Furthermore, it was also determined whether the rate of margin positivity was decreased by re-excision based on SMMG evaluation.

\section{Results}

Patient characteristics. A total of 174 women who underwent BCS were reviewed. The mean age was 54.7 years (range, 26-84 years). The median tumor size was $1.6 \mathrm{~cm}$ (range, $0-4.5 \mathrm{~cm}$ ) and 132 of the masses were palpable (75.9\%). The MMG findings revealed that 51 patients had microcalcifications (29.3\%). The histopathological diagnosis of the patients was as follows: DCIS, $\mathrm{n}=32$ (18\%); invasive ductal carcinoma (IDC), $\mathrm{n}=121(69 \%)$; invasive lobular carcinoma, $\mathrm{n}=10(6 \%)$; and others, $\mathrm{n}=11(6 \%)$ (Table II).
Table I. Evaluation of intraoperative specimen mammography by two clinicians.

\begin{tabular}{lcc}
\hline Clinician A & Clinician B & Final evaluation \\
\hline Positive & Close & Positive \\
Positive & Negative & Positive \\
Positive & Undetected & Positive \\
Close & Close & Positive \\
Close & Undetected & Positive \\
Close & Negative & Negative \\
Negative & Undetected & Negative \\
\hline
\end{tabular}

Table II. Radiological and pathological patient characteristics $(n=174)$.

\begin{tabular}{lc}
\hline Characteristics & $\mathrm{n}(\%)$ \\
\hline Age (years) & \\
$<50$ & $113(65)$ \\
$\geq 50$ & $61(35)$ \\
Palpability & \\
Non-palpable & $42(24)$ \\
Palpable & $132(76)$ \\
Tumor stage & \\
T1 & $97(56)$ \\
T2 & $42(24)$ \\
T3-4 & $3(2)$ \\
Mammographic image & \\
Calcifications only & $18(10)$ \\
Calcifications + others & $33(19)$ \\
Others & $110(63)$ \\
None & $13(8)$ \\
Histology & \\
Invasive ductual carcinoma & $121(70)$ \\
Invasive lobular carcinoma & $10(6)$ \\
Ductal carcinoma in situ & $32(18)$ \\
Others & $11(6)$ \\
\hline
\end{tabular}

Association between histological margin status and re-excision. The association between the histological margin status and re-excision is shown in Fig. 1. Re-excision was performed intraoperatively in 24 cases following positive margin identification by SMMG. Of these 24 cases, 5 (20.8\%) still had positive margins following re-excision. However, 150 cases did not undergo re-excision and 30 of those cases had positive margins.

On radiological evaluation, 17 cases $(10 \%)$ had a positive margin, 146 cases $(84 \%)$ had a negative margin and 11 cases (6\%) had undetected lesions. Histological evaluation of the margins revealed 35 positive $(20 \%)$ and 139 negative $(80 \%)$ cases. The intraoperative re-excision cases were excluded from this analysis, as they did not undergo a second SMMG and the histological margin status was definitively determined after 


\section{4 patients with breast cancer}

underwent BCS

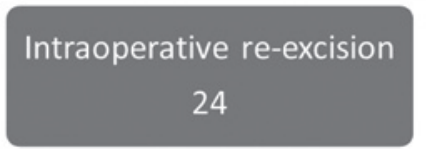

No additional treatment

150

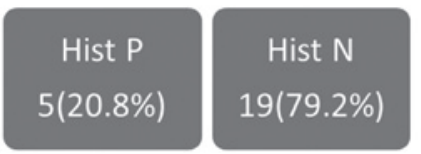

Hist P

$30(20.0 \%)$

Hist $N$

$120(80.0 \%)$

Figure 1. Association between histological margin status and re-excision BCS, breast-conserving surgery. Hist P, positive histological margin; Hist N, negative histological margin.

Table III. SMMG results and histological findings.

\begin{tabular}{lccc}
\hline & \multicolumn{2}{c}{ Margin by histology (n) } & \\
\cline { 2 - 3 } Margin by imaging & Positive & Negative & Total (n) \\
\hline Positive (n) & 6 & 6 & 12 \\
Negative (n) & 23 & 106 & 129 \\
Total (n) & 29 & 112 & 141 \\
\hline
\end{tabular}

Intraoperative re-excision cases and lesions undetected on SMMG were excluded. SMMG, specimen mammography.

the first excision. Moreover, the lesions that were not detected by SMMG were also excluded.

Association between SMMG and histopathological findings. Finally, a total of 141 cases were analyzed and the association between SMMG and histopathological findings was evaluated (Table III). A total of 23 false-negatives and 6 false-positives were identified. The sensitivity and specificity of SMMG margin assessment for primary breast cancer were 20.6 and $94.6 \%$, respectively. The positive predictive value was $50 \%$ and the negative predictive value was $82.2 \%$.
Subgroup analysis. Finally, a subgroup analysis was performed (Table IV). The number of true positive cases was four in the microcalcification group, five in the non-palpable group, one in group of patients aged $\geq 50$ years and two in the IDC group. The sensitivity and specificity for patients exhibiting microcalcifications $(n=37$ after exclusion of 24 cases with intraoperative re-excision and of nine cases in which intra-operative SMMG did not display lesions) were 44.4 and $89.2 \%$, for patients aged $\geq 50$ years they were 6.6 and $94.7 \%$, and for patients with non-palpable lesions they were 50 and $91.6 \%$, respectively. Following histopathological classification of all the cases, the sensitivity and specificity of SMMG for IDC were 10 and $95.1 \%$, and for DCIS they were 75 and $100 \%$, respectively. Local recurrence only developed in 2 cases over a median follow-up of 5.4 years; both cases had negative margins in the primary operation.

\section{Discussion}

When performing BCS, the main objective is to obtain clear resection margins, as a positive margin presents a major risk of local relapse $(14,15)$. Intraoperative margin assessment in BCS is crucial in order to avoid secondary surgery. The rates of positive margins on the first attempt of BCS or lumpectomy have been reported to be as high as $55-68 \%$ in the USA $(16,17)$. Despite significant improvements in breast imaging, the rate of positive margins remains high.

SMMG is convenient, easy and cost-effective, and has been used for margin assessment in our institution. Several studies have demonstrated that intraoperative margin assessment using frozen sections of the surgical specimen is useful (12,18-20). However, histological intraoperative margin assessment may be associated with prolonged operative time and requires pathological expertise.

Bathla et al (21) reported that $99 \%$ of the patients ultimately deemed as BCS candidates underwent successful breast conservation. In that study, $14.7 \%$ of the patients underwent BCS as the secondary surgery (21). In the present study, only 1 patient $(0.5 \%)$ underwent BCS as a second surgical intervention, while several patients opted for mastectomy as the second surgery. The efficacy of bidirectional SMMG was previously reported $(21,22)$. In the present study, however, SMMG was not performed in two directions, as the BCS procedure involved columnar resection between the skin and the pectoralis fascia,

Table IV. Sensitivity and specificity of each subgroup.

\begin{tabular}{|c|c|c|c|c|c|c|}
\hline \multirow[b]{2}{*}{ Variables } & \multicolumn{2}{|c|}{ Imaging (n) } & \multicolumn{2}{|c|}{ Histology (n) } & \multirow[b]{2}{*}{ Sensitivity (\%) } & \multirow[b]{2}{*}{ Specificity (\%) } \\
\hline & Positive & Negative & Positive & Negative & & \\
\hline Calcifications (n=37) & 7 & 30 & 9 & 28 & 44.4 & 89.2 \\
\hline Non-palpable $(\mathrm{n}=34)$ & 7 & 27 & 10 & 24 & 50.0 & 91.6 \\
\hline Age $\geq 50$ years $(n=91)$ & 5 & 86 & 15 & 76 & 6.6 & 94.7 \\
\hline \multicolumn{7}{|l|}{ Histology } \\
\hline IDC ( $\mathrm{n}=103)$ & 6 & 97 & 20 & 83 & 10.0 & 95.1 \\
\hline DCIS (n=22) & 3 & 19 & 4 & 18 & 75.0 & 100.0 \\
\hline
\end{tabular}

IDC, invasive ductual carcinoma; DCIS, ductal carcinoma in situ. 
rather than a spherical resection, which is common in western countries.

Intraoperative SMMG was not proven to be useful in the present study. There are two possible explanations: First, a simple comparison between the pathological results and SMMG findings was performed with the aim of determining whether the resection margins may be reliably evaluated by SMMG. However, as shown in Table III, the sensitivity of SMMG was very low, particularly for patients aged $\geq 50$ years $(6.6 \%)$. Although it was hypothesized that the density of the breast affected the evaluation by SMMG, this hypothesis was proven to be false by these results. In fact, in certain cases a lesion was detected by SMMG that was not detected on preoperative MMG. Furthermore, the majority of the cases in which a lesion was undetectable, were those of patients aged $\geq 50$ years. In other subgroups, e.g. patients with microcalcifications or non-palpable lesions, the sensitivity of SMMG was also low, whereas the sensitivity in DCIS cases $(n=22)$ was marginally high at $75 \%$ and the positive predictive value was $100 \%$. This result was also unexpected, but it may not be reliable due to the small number of cases. Second, the margin-positive rate in patients undergoing re-excision was $20.8 \%$ and in those without re-excision $20.0 \%$ (Fig. 1). Thus, the margin-positive rate could not be reduced despite re-excision based on SMMG, suggesting that SMMG was not useful.

Of note, in the 6 cases in which breast cancer was diagnosed by stereotactic biopsy, the histopathological results were in complete accord with the SMMG findings, indicating that SMMG is likely useful in such cases.

However, the present study had certain limitations. First, 24 cases with re-excision were excluded from the evaluation of histology and radiology findings. The final resection margin and histopathological results could not be compared, since not all of the re-excision cases underwent a second SMMG. Second, there were no exact criteria for the identification of positive margins on radiological examination. To overcome these limitations, a prospective study with strictly defined criteria and a standard procedure is required to determine the usefulness of SMMG.

In conclusion, the usefulness of intraoperative SMMG was not proven in this study. However, this procedure is likely to be useful in selected cases, particularly those with DCIS.

\section{References}

1. Lovrics PJ, Cornacchi SD, Farrokhyar F, Garnett A, Chen V, Franic S and Simunovic M: The relationship between surgical factors and margin status after breast-conservation surgery for early stage breast cancer. Am J Surg 197: 740-746, 2009.

2. Fisher B, Anderson S, Bryant J, et al: Twenty-year follow-up of a randomized trial comparing total mastectomy, lumpectomy, and lumpectomy plus irradiation for the treatment of invasive breast cancer. N Engl J Med 347: 1233-1241, 2002.

3. Povoski SP, Jimenez RE, Wang WP and Xu RX: Standardized and reproducible methodology for the comprehensive and systematic assessment of surgical resection margins during breast-conserving surgery for invasive breast cancer. BMC Cancer 9: 254, 2009.

4. Blichert-Toft M, Brincker H, Andersen JA, et al: A Danish randomized trial comparing breast-preserving therapy with mastectomy in mammary carcinoma. Preliminary results. Acta Oncol 27: 671-677, 1988.

5. van Dongen JA, Voogd AC, Fentiman IS, et al: Long-term results of a randomized trial comparing breast-conserving therapy with mastectomy: European Organization for Research and Treatment of Cancer 10801 trial. J Natl Cancer Inst 92: 1143-1150, 2000.
6. van Dongen JA, Bartelink H, Fentiman IS, Lerut T, Mignolet F, Olthuis G, van der Schueren E, Sylvester R, Tong D, Winter J, et al: Factors influencing local relapse and survival and results of salvage treatment after breast-conserving therapy in operable breast cancer: EORTC trial 10801, breast conservation compared with mastectomy in TNM stage I and II breast cancer. Eur J Cancer 28A: 801-805, 1992.

7. Park CC, Mitsumori M, Nixon A, Recht A, Connolly J, Gelman R, Silver B, Hetelekidis S, Abner A, Harris JR and Schnitt SJ: Outcome at 8 years after breast-conserving surgery and radiation therapy for invasive breast cancer: Influence of margin status and systemic therapy on local recurrence. J Clin Oncol 18: 1668-1675, 2000.

8. Veronesi U, Luini A, Galimberti V and Zurrida S: Conservation approaches for the management of stage I/II carcinoma of the breast: Milan Cancer Institute trials. World J Surg 18: 70-75, 1994.

9. Clarke M, Collins R, Darby S, Davies C, Elphinstone P, Evans V, Godwin J, Gray R, Hicks C, James S, et al: Effects of radiotherapy and of differences in the extent of surgery for early breast cancer on local recurrence and 15-year survival: An overview of the randomised trials. Lancet 366: 2087-2106, 2005.

10. Moran MS, Schnitt SJ, Giuliano AE, Harris JR, Khan SA, Horton J, Klimberg S, Chavez-MacGregor M, Freedman G, Houssami N, et al; Society of Surgical Oncology; American Society for Radiation Oncology: Society of Surgical Oncology-American Society for Radiation Oncology consensus guideline on margins for breast-conserving surgery with whole-breast irradiation in stages I and II invasive breast cancer. J Clin Oncol 32: 1507-1515, 2014.

11. Ichihara S, Suzuki H, Kasami M, Aoyama H, Sato Y, Oiwa M, Kurokawa K and Endo T: A new method of margin evaluation in breast conservation surgery using an adjustable mould during fixation. Histopathology 39: 85-92, 2001.

12. Wright MJ, Park J, Fey JV, Park A, O'Neill A, Tan LK, Borgen PI, Cody HS III, Van Zee KJ and King TA: Perpendicular inked versus tangential shaved margins in breast-conserving surgery: Does the method matter? J Am Coll Surg 204: 541-549, 2007.

13. Guidi AJ, Connolly JL, Harris JR and Schnitt SJ: The relationship between shaved margin and inked margin status in breast excision specimens. Cancer 79: 1568-1573, 1997.

14. Houssami N, Macaskill P, Marinovich ML, Dixon JM, Irwig L, Brennan ME and Solin LJ: Meta-analysis of the impact of surgical margins on local recurrence in women with early-stage invasive breast cancer treated with breast-conserving therapy. Eur J Cancer 46: 3219-3232, 2010.

15. Singletary SE: Surgical margins in patients with early-stage breast cancer treated with breast conservation therapy. Am J Surg 184: 383-393, 2002.

16. Christy CJ, Thorsteinsson D, Grube BJ, Black D, Abu-Khalaf M, Chung GG, DiGiovanna MP, Miller K, Higgins SA, Weidhaas J, et al: Preoperative chemotherapy decreases the need for re-excision of breast cancers between 2 and $4 \mathrm{~cm}$ diameter. Ann Surg Oncol 16: 697-702, 2009.

17. O'Sullivan MJ, Li T, Freedman G and Morrow M: The effect of multiple reexcisions on the risk of local recurrence after breast conserving surgery. Ann Surg Oncol 14: 3133-3140, 2007.

18. Cabioglu N, Hunt KK, Sahin AA, Kuerer HM, Babiera GV, Singletary SE, Whitman GJ, Ross MI, Ames FC, Feig BW, et al: Role for intraoperative margin assessment in patients undergoing breast-conserving surgery. Ann Surg Oncol 14: 1458-1471, 2007.

19. Sauter ER, Hoffman JP, Ottery FD, Kowalyshyn MJ, Litwin S and Eisenberg BL: Is frozen section analysis of reexcision lumpectomy margins worthwhile? Margin analysis in breast reexcisions. Cancer 73: 2607-2612, 1994.

20. Cendán JC, Coco D and Copeland EM III: Accuracy of intraoperative frozen-section analysis of breast cancer lumpectomy-bed margins. J Am Coll Surg 201: 194-198, 2005.

21. Bathla L, Harris A, Davey M, Sharma P and Silva E: High resolution intra-operative two-dimensional specimen mammography and its impact on second operation for re-excision of positive margins at final pathology after breast conservation surgery. Am J Surg 202: 387-394, 2011.

22. Fouché CJ, Tabareau F, Michenet P, Lebas P and Simon EG: Specimen radiography assessment of surgical margins status in subclinical breast carcinoma: A diagnostic study. J Gynecol Obstet Biol Reprod (Paris) 40: 314-322, 2011 (In French). 